\title{
Safety and efficacy of the European Society of Cardiology 0/1-hour algorithm for diagnosis of myocardial infarction: systematic review and meta- analysis
}

\author{
Cho-Han Chiang, ${ }_{1}^{1}$ Cho-Hung Chiang, ${ }^{2}$ Gin Hoong Lee, ${ }^{1}$ Weng-Tein Gi, ${ }^{3}$ Yuan-Kun Wu, ${ }^{4}$ \\ Sih-Shiang Huang, ${ }^{4}$ Yee Hui Yeo, ${ }^{5}$ Evangelos Giannitsis, ${ }^{3}$ Chien-Chang Lee (D) ${ }^{6,7,8}$
}

- Additional material is published online only. To view please visit the journal online (http://dx.doi.org/10.1136/ heartjnl-2019-316343).

For numbered affiliations see end of article.

\section{Correspondence to} Professor Chien-Chang Lee, MD, ScD; Health Data Science Research Group; The Centre for Intelligent Healthcare; Department of Emergency Medicine, National Taiwan University Hospital, Taipei 100, Taiwan; cclee100@gmail.com

C-HC, C-HC and GHL contributed equally.

Received 27 November 2019 Revised 21 February 2020 Accepted 25 February 2020 Published Online First 3 April 2020

\section{Linked}

- http://dx.doi.org/10.1136/ heartjnl-2020-316811

Check for updates

(c) Author(s) (or their employer(s)) 2020. No commercial re-use. See rights and permissions. Published by BMJ.

To cite: Chiang $\mathrm{C}-\mathrm{H}$ Chiang C-H, Lee GH, et al. Heart 2020;106:985-991.

\section{ABSTRACT}

Objective The European Society of Cardiology (ESC) $0 / 1$ hour algorithm has been primarily validated in Europe, America and Australasia with less knowledge of its performance outside of these settings. We aim to evaluate the performance of the ESC 0/1 hour algorithm across different contexts.

Methods We searched PubMed, Embase, Scopus, Web of Science and the Cochrane Central Register of Controlled Trials for relevant studies published between 1 January 2008 and 31 May 2019. The primary outcome was index myocardial infarction and the secondary outcome was major adverse cardiac event or mortality. A bivariate random-effects meta-analysis was used to derive the pooled estimate of each outcome.

Results A total of 11014 patients from 10 cohorts were analysed for the primary outcome. The algorithm based on high-sensitivity cardiac troponin (hs-cTn) T (Roche), hs-cTnl (Abbott) and hs-cTnl (Siemens) had pooled sensitivity of $98.4 \%(95 \% \mathrm{Cl}=95.1 \%$ to $99.5 \%)$, $98.1 \%(95 \% \mathrm{Cl}=94.6 \%$ to $99.3 \%)$ and $98.7 \%(95 \%$ $\mathrm{Cl}=97.3 \%$ to $99.3 \%)$, respectively. The algorithm based on hs-cTnT (Roche) and hs-cTnl (Siemens) had pooled specificity of $91.2 \%(95 \% \mathrm{Cl}=86.0 \%$ to $94.6 \%)$ and $95.9 \%(95 \% \mathrm{Cl}=94.1 \%$ to $97.2 \%)$, respectively. Among patients in the rule-out category, the pooled mortality rate at 30 days and at 1 year was $0.1 \%(95 \%$ $\mathrm{Cl}=0.0 \%$ to $0.4 \%)$ and $0.8 \%(95 \% \mathrm{Cl}=0.5 \%$ to $1.2 \%)$, respectively. Among patients in the observation zone, the pooled mortality rate was $0.7 \%(95 \% \mathrm{Cl}=0.3 \%$ to $1.2 \%)$ at 30 days but increased to $8.1 \%(95 \% \mathrm{Cl}=6.1 \%$ to $10.4 \%$ ) at 1 year, comparable to the mortality rate in the rule-in group.

Conclusion The ESC 0/1 hour algorithm has high diagnostic accuracy but may not be sufficiently safe if the $1 \%$ miss-rate for myocardial infarction is desired.

PROSPERO registration

number CRD42019142280.

\section{INTRODUCTION}

Chest pain and associated symptoms constitute a significant proportion of the emergency department (ED) visits. ${ }^{12}$ However, only 10\%-20\% of the patients presenting with these complaints are eventually diagnosed with acute myocardial infarction (AMI). ${ }^{3}$ Timely diagnosis of AMI is essential because early revascularisation with an evidencebased care bundle can decrease mortality. ${ }^{4}$

The recent introduction of high-sensitivity cardiac troponin (hs-cTn) assays has allowed the development of several rapid triage protocols, including the European Society of Cardiology (ESC) 0/1 hour algorithm. ${ }^{5}$ This approach combines a very low hs-cTn at initial ED presentation and dynamic change of hs-cTn values between 0 and 1 hour to triage patients into rule-out, observation and rule-in categories, allowing clinical decision making in just over 2-3 hours.

Even though the ESC 0/1 hour algorithm has been validated in a large number of prospective studies $^{6-12}$, use of this algorithm has been limited to less than $15 \%$ of the hospital institutions globally. ${ }^{13}$ Because the algorithm has primarily been evaluated in Europe, America and Australasia, ${ }^{6-9}$ there are some concerns about its safety outside of these settings. Furthermore, the reported diagnostic accuracies seem to be discrepant across different population cohorts. ${ }^{14-16}$

Previous meta-analyses have focused on the utility of a single hs-cTn test at ED presentation to rule out AMI. ${ }^{17-22}$ The diagnostic accuracy of the $0 / 1$ hour delta change of hs-cTn, which is central to the ESC algorithm, has not been evaluated. Importantly, none of these studies reported on the prognostic significance of the hs-cTn assays based on the respective triage categories. The outcome of patients that fall into the rule-out and observation group is of great importance for clinical decision making.

To address these knowledge gaps, we conducted a systematic review and meta-analysis to investigate the accuracy estimates and prognostic values of the ESC 0/1 hour algorithm.

\section{METHODS}

This study was conducted in accordance with the Preferred Reporting Items for Systematic Review and Meta-analysis of Diagnostic Test Accuracy Studies $^{23}$ and Meta-analyses Of Observational Studies in Epidemiology guidelines. ${ }^{24}$ The review protocol was registered on the PROSPERO database. Patients or the public were not involved in the design, or conduct, or reporting, or dissemination plans of this research. 


\section{Search strategy}

We searched PubMed and Embase for studies published between 1 January 2008 (when the first hs-cTnI assay was released) and 31 May 2019 using a protocol (online supplementary table 1) that incorporated keywords myocardial infarction, troponin, $0 / 1$ hour algorithm and emergency department. References for original and review articles were manually searched. An updated search on Scopus, Web of Science and the Cochrane Central Register of Controlled Trials was performed to ensure the completeness of data search. There were no restrictions on language. Articles that were published in languages other than English were translated and interpreted using Google translation.

\section{Study selection}

Three reviewers (C-HC, C-HC, GHL) independently screened the titles and abstracts to identify potentially relevant studies. Full texts of relevant publications were screened independently by three reviewers (C-HC, C-HC, GHL). A fourth reviewer (C-CL) confirmed the inclusion or exclusion of the studies.

Eligible studies were prospective cohort studies that evaluated the diagnostic accuracy of the ESC $0 / 1$ hour algorithm in patients presenting to the ED with suspected non-ST elevation myocardial infarction (NSTEMI) or acute coronary syndrome (ACS). Studies that evaluated the high-sensitivity cardiac troponin $\mathrm{T}$ (hs-cTnT) (Roche) and high-sensitivity cardiac troponin I (hscTnI) (Abbott) assays were included. ${ }^{5}$ Studies that assessed the hs-cTnI (Diemension Vista) assay were excluded. Studies on other assays that were not yet recommended by the 2015 ESC guidelines were included if there were three or more cohorts available.

Studies should use myocardial infarction as the primary endpoint based on the contemporary universal definition. ${ }^{25}$ Studies should exclude patients with initial diagnosis of STEMI, or include sub-group data on only patients with NSTEMI. Studies that used major adverse cardiovascular events (MACE) or mortality as the primary endpoint were also included for analysis of the safety profile. Conference papers were only included if there were no previous original articles published. For cohorts with multiple publications that have identical study endpoints and overlapping recruitment periods, only the study with the largest sample size or most complete datasets was included (online supplementary methods).

\section{Data extraction}

Three reviewers (C-HC, C-HC, GHL) independently abstracted data on study demographics, and both primary and secondary outcomes. The primary endpoint was index admission AMI based on the universal definition of AMI, and the secondary endpoints were death and occurrence of MACE.

\section{Quality assessment}

The methodological quality of included studies was assessed by two reviewers (C-HC and C-HC) using the Quality Assessment of Diagnostic Accuracy Studies-2 tool (online supplementary tables 2 and 3). ${ }^{26}$ Any discrepancies were resolved through discussion with a third reviewer (GHL).

\section{Data synthesis and analysis}

Since the ESC 0/1 hour algorithms categorised patients into rule-out, observation and rule-in groups, we calculated pooled sensitivity, negative predictive value (NPV), and negative likelihood ratios (LR-) for the rule-out group, and pooled specificity, positive predictive value (PPV), and positive likelihood ratios

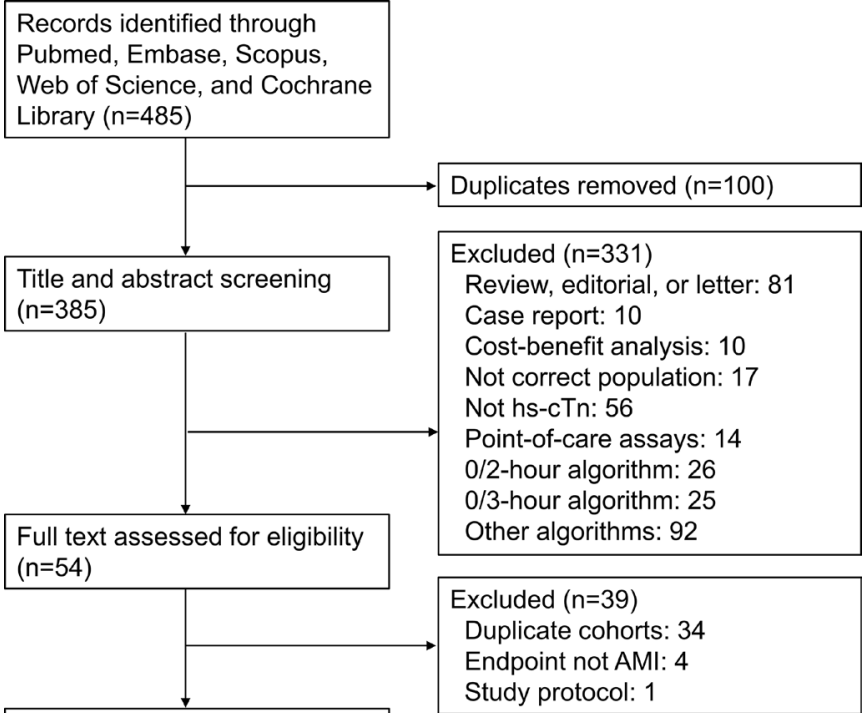

Studies included for analysis $(n=15)$

Figure 1 Flowchart describing the process of study selection. AMI, acute myocardial infarction; hs-cTn, high-sensitivity cardiac troponin.

$(\mathrm{LR}+)$ for the rule-in group separately. We computed triage efficacy as the proportion of patients that were placed in the respective triage groups. Accuracy estimates were pooled using bivariate random-effects regression models. The between-study variation was quantified by $\mathrm{I}^{2}$ statistics. The presence and effect of publication bias were examined using the Deek's test. ${ }^{27} \mathrm{We}$ performed a meta-analysis of proportions to compare the rate of adverse events and proportion of patients in different triage groups. We conducted sensitivity analyses based on risks of bias. Continuous variables were described as mean or median with 95\% CI or IQR, categorical variables as numbers and percentages. All analyses were calculated by the 'midas,' 'metabias,' 'Fagani' and 'metaprop' package in STATA V.14.0 or R software V.3.5.3.

\section{RESULTS}

Initial electronic database searches yielded 385 hits. After title and abstract screening, 54 studies were eligible for full-text review. After full-text review, a total of 15 studies were included for analysis(figure 1 ) 679 14-16 28-36.

\section{Characteristics of included studies}

Overall, 64\% (range, 40\% to 70\%) of 11014 participants were male, with a mean age of 61 years (range, 55-72 years). The prevalence of AMI ranged from $2 \%$ to $32 \%$, with an overall pooled prevalence of $16 \%$. The time from ED presentation to first sample collection ranged from $14.9 \mathrm{~min}$ to $28 \mathrm{~min}$, and the time from first to second sample collection ranged from 60 min to $65 \mathrm{~min}$ (table 1). The key characteristics and details of accuracy estimates of all included studies are described in online supplementary tables 4 to 8 .

\section{Quality assessment}

All studies prospectively recruited patients presenting to the ED with symptoms suggestive of AMI. Some studies enrolled consecutive or random samples of patients (online supplementary figure 1 and table 3 ). Most studies used pre-specified cutoff points to classify patients; three studies derived the $0 / 1$ hour algorithm. ${ }^{6729}$ In all studies, AMI was defined according to the 
Table 1 Characteristics of studies included in the systematic review

\begin{tabular}{|c|c|c|c|c|c|c|c|c|c|}
\hline Cohort & Study (author) & $\begin{array}{l}\text { Mean age }(95 \% \\
\mathrm{Cl})\end{array}$ & $\begin{array}{l}\text { Sample } \\
\text { size }\end{array}$ & $\begin{array}{l}\text { Male } \\
(\%)\end{array}$ & $\begin{array}{l}\text { AMI } \\
\text { prevalence } \\
(\%)\end{array}$ & $\begin{array}{l}\text { Median } \\
\text { presentation } \\
\text { time (min) }\end{array}$ & $\begin{array}{l}\text { Median time from } \\
\text { presentation to } \\
\text { first sample (min) }\end{array}$ & $\begin{array}{l}\text { Median time } \\
\text { between first } \\
\text { and second } \\
\text { sample (min) }\end{array}$ & hs-cTn assay \\
\hline $\begin{array}{l}\text { APACE } \\
\text { (derivation) }\end{array}$ & Gimenez et al ${ }^{7}$ & 62 (49 to 62$)$ & 905 & 69 & 21 & NA & NA & NA & hs-cTnl-Abbott \\
\hline APACE-BACC & Twerenbold et $a^{28}$ & 62 (50 to 74 ) & 4368 & 67 & 17 & NA & 0 & $60(59-68)$ & $\begin{array}{l}\text { hs-cTnT-Roche and } \\
\text { hs-cTnl-Abbott }\end{array}$ \\
\hline HIGHSTEAC & Chapman et al ${ }^{\beta 0} 31$ & $62 \pm 14.2$ & 406 & 61 & 8 & 199 & 28 & $65(60-73)$ & $\begin{array}{l}\text { hs-cTnl-Abbott and } \\
\text { hs-cTnl-Siemens }\end{array}$ \\
\hline Barcelona & $\begin{array}{l}\text { Duran-Cambra } \\
\text { et } \mathrm{al}^{16}\end{array}$ & $68 \pm 15$ & 187 & 64 & 13 & NA & NA & NA & hs-cTnT-Roche \\
\hline Parkland & Vigen et $a /^{15}$ & 55 & 536 & 56 & 2 & NA & NA & NA & hs-cTnT-Roche \\
\hline Fuwai & Lin et $\left.a\right|^{\beta 6}$ & 59.5 (58.2 to 60.8$)$ & 283 & NA & 32 & NA & NA & NA & hs-cTnl-Abbott \\
\hline Bangkok* & $\begin{array}{l}\text { Ruangsomboon } \\
\text { et } a^{\beta 2}\end{array}$ & $67 \pm 14.2$ & 65 & 40 & NA & 186 & 14.9 & NA & hs-cTnT-Roche \\
\hline Lund* & Mokhtari et al ${ }^{33}$ & $61 \pm 7.5$ & 1038 & 54 & NA & NA & NA & NA & hs-cTnT-Roche \\
\hline
\end{tabular}

${ }^{*}$ These cohorts were included for analysis of secondary outcome, that is, major adverse cardiac events or mortality.

AMI, acute myocardial infarction; APACE, advantageous predictors of acute coronary syndromes evaluation; BACC, biomarkers in acute cardiac care; HIGHSTEACS, high-sensitivity troponin in the evaluation of patients with acute coronary syndrome; HIGH-US, high-sensitivity cardiac troponin I assays in the United States; hs-cTn, high-sensitivity cardiac troponin; NA, Not available; REACTION-US, Rapid evaluation of acute myocardial infarction in the United States; TRAPID, the high sensitivity cardiac troponin T assay for rapid rule-out of acute myocardial infarction.

Global Task Force and adjudicated independently by two or three physicians. ${ }^{25}$ In three studies, researchers who adjudicated the outcomes were blinded to the results of the index test. ${ }^{914} 33$ Six studies that did not employ blinding during the adjudication process also used hs-cTn clinically for adjudication of MI. ${ }^{6728-31}$ Several studies did not include all eligible patients for analysis because of missing data, most commonly due to missing samples at 1 hour.

\section{Rule-out performance}

Based on hs-cTnT (Roche), the algorithm ruled out $55 \%$ of the patients as low risk for AMI, with a pooled sensitivity of $98.4 \%$ $\left(95 \% \mathrm{CI}=95.1 \%\right.$ to $\left.99.5 \% ; \mathrm{I}^{2}=29 \%\right)$ and NPV of $99.6 \%(95 \%$ $\mathrm{CI}=99.0 \%$ to $99.9 \%$ ) (figure 2 and online supplementary table 9). Similarly for hs-cTnI (Abbott) and hs-cTnI (Siemens), the algorithm ruled out more than $50 \%$ of the patients as low risk with pooled sensitivities of $98.1 \%$ (95\% CI $=94.6 \%$ to $99.3 \%$; $\left.\mathrm{I}^{2}=25 \%\right)$ and $98.7 \%\left(95 \% \mathrm{CI}=97.3 \%\right.$ to $\left.99.3 \% ; \mathrm{I}^{2}=17 \%\right)$, and NPVs of $99.3 \%(95 \% \mathrm{CI}=95.9 \%-99.9 \%)$ and $99.6 \%(95 \%$ $\mathrm{CI}=99.2 \%$ to $99.8 \%$ ), respectively.

\section{Rule-in performance}

Based on hs-cTnT (Roche), the algorithm ruled in 18\% of the patients as high risk for AMI, with a pooled specificity of $91.2 \%$ (95\% CI $=86.0 \%$ to $\left.94.6 \% ; \mathrm{I}^{2}=11 \%\right)$ and PPV of $51.3 \%(95 \%$ $\mathrm{CI}=30.8 \%$ to $71.3 \%$ ) (figure 3 and online supplementary table 10). For hs-cTnI (Siemens), the algorithm ruled in $14 \%$ of the patients with a higher specificity at $95.9 \%(95 \% \mathrm{CI}=94.1 \%$ to 97.2\%; $\mathrm{I}^{2}=3 \%$ ). Hs-cTnI (Abbott) could not be pooled owing to insufficient studies.

\section{Prognosis of patients in different triage groups}

For patients in the rule-out group, the pooled 30-day mortality rate was $0.1 \%\left(95 \% \mathrm{CI}=0.0 \%\right.$ to $\left.0.4 \% ; \mathrm{I}^{2}=0 \%\right)$ and increased slightly to $0.8 \%\left(95 \% \mathrm{CI}=0.5 \%\right.$ to $\left.1.2 \% ; \mathrm{I}^{2}=0 \%\right)$ at 1 year (figure 4 and online supplementary table 11). For patients in the observation group, the pooled 30-day mortality rate was $0.7 \%\left(95 \% \mathrm{CI}=0.3 \%\right.$ to $\left.1.2 \% ; \mathrm{I}^{2}=0 \%\right)$, increasing substantially to $8.1 \%\left(95 \% \mathrm{CI}=6.1 \%\right.$ to $\left.10.4 \% ; \mathrm{I}^{2}=0 \%\right)$ at 1 year. For the rule-in group, the pooled 30-day mortality rate was $1.8 \%$ ( $95 \% \mathrm{CI}=0.4 \%$ to $\left.4.2 \% ; \mathrm{I}^{2}=0 \%\right)$, increasing to $10.0 \%(95 \%$ $\mathrm{CI}=7.8 \%$ to $\left.12.4 \% ; \mathrm{I}^{2}=0 \%\right)$ at 1 year.

\section{Sensitivity analysis}

In a sensitivity analysis pooling all studies eligible for estimation of the $0 / 1$ hour algorithm, irrespective of assay or quality assessment, the sensitivity of the algorithm in ruling out AMI was $98.3 \%$ (95\% CI=97.0\% to $99.1 \%$ ) (online supplementary figure $2 \mathrm{~A}$ ), and the specificity of the algorithm in ruling in AMI was $93.4 \%$ (95\% CI $=90.6 \%$ to $95.4 \%$ ) (online supplementary figure $2 \mathrm{~B}$ ). Three cohorts were the derivation cohorts for the $0 / 1$ hour algorithm (one for each assay) and were subjected to high risk of bias. After these cohorts were removed, the remaining validation cohorts had a sensitivity of $98.1 \%(95 \% \mathrm{CI}=96.3 \%$ to $99.0 \%$ ) (online supplementary figure $2 \mathrm{C}$ ) and specificity of $93.2 \%(95 \% \mathrm{CI}=89.2 \%$ to $95.7 \%$ ) (online supplementary figure 2D), comparable to that in the overall pooled analysis.

\section{DISCUSSION}

In this meta-analysis, the ESC 0/1 hour algorithm ruled out more than $50 \%$ of the patients presenting to the ED with chest pain as low risk for AMI. The algorithm has a good sensitivity and low rates of adverse cardiac events in the rule-out group, and 


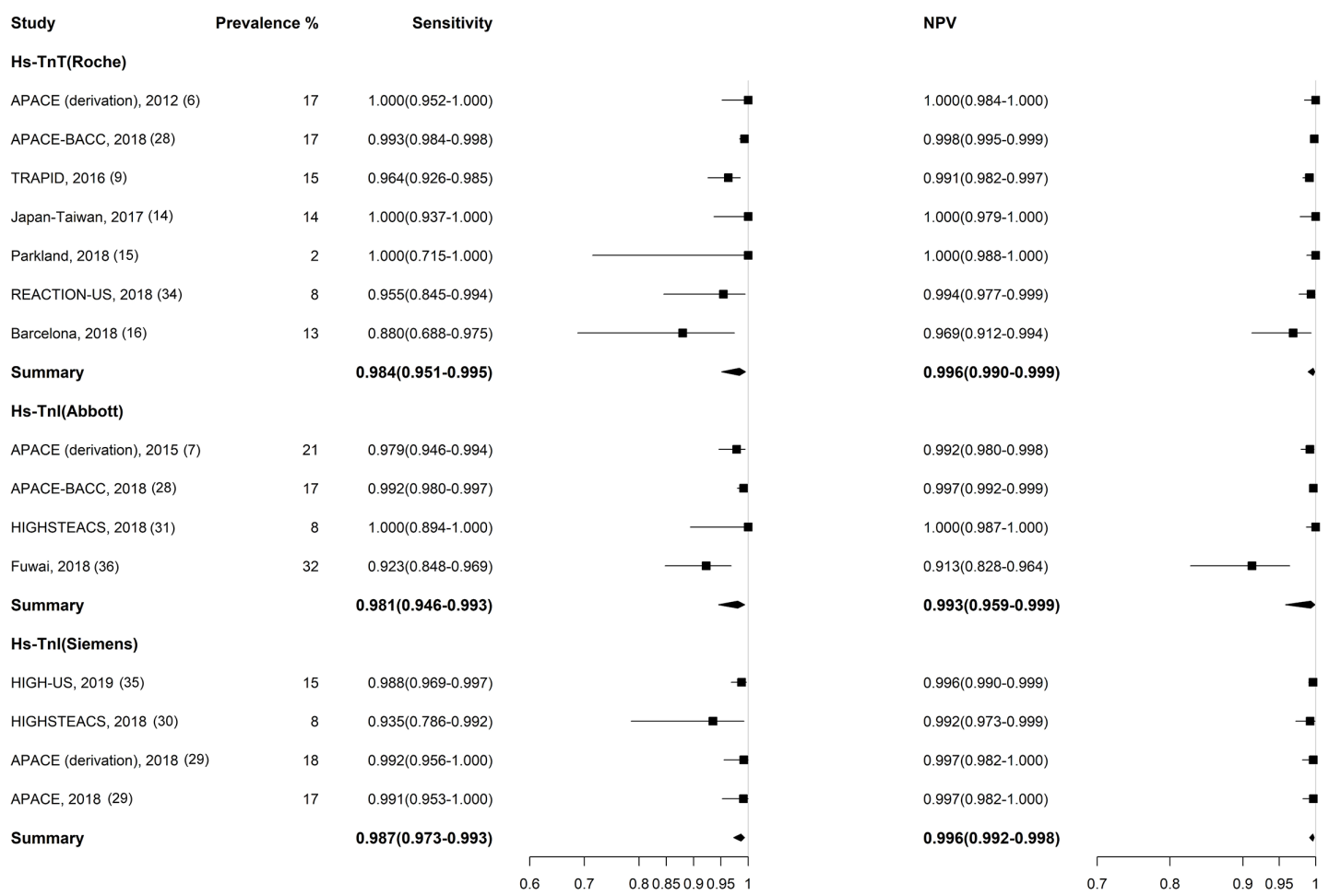

Figure 2 Forest plot for acute myocardial infarction and summary estimates for sensitivity and NPV. Note: Reichlin et al ${ }^{6}$ (derivation cohort for hsCTnT (Roche)) set an optimal threshold for a 100\% sensitivity and NPV. Rubini et al (derivation cohort for hs-cTnl (Abbott)) set an optimal threshold for a $99 \%$ sensitivity and $95 \%$ NPV. Boeddinghaus et a ${ }^{29}$ (derivation cohort for hs-cTnl (Siemens)) set an optimal threshold for a $99 \%$ sensitivity and NPV. APACE, advantageous predictors of acute coronary syndromes evaluation; BACC, biomarkers in acute cardiac care; HIGHSTEACS, high-sensitivity troponin in the evaluation of patients with acute coronary syndrome; HIGH-US, high-sensitivity cardiac troponin I assays in the United States; NPV, negative predictive value; REACTION-US, rapid evaluation of acute myocardial infarction in the United States; TRAPID, the high sensitivity cardiac troponin T assay for rapid rule-out of acute myocardial infarction.

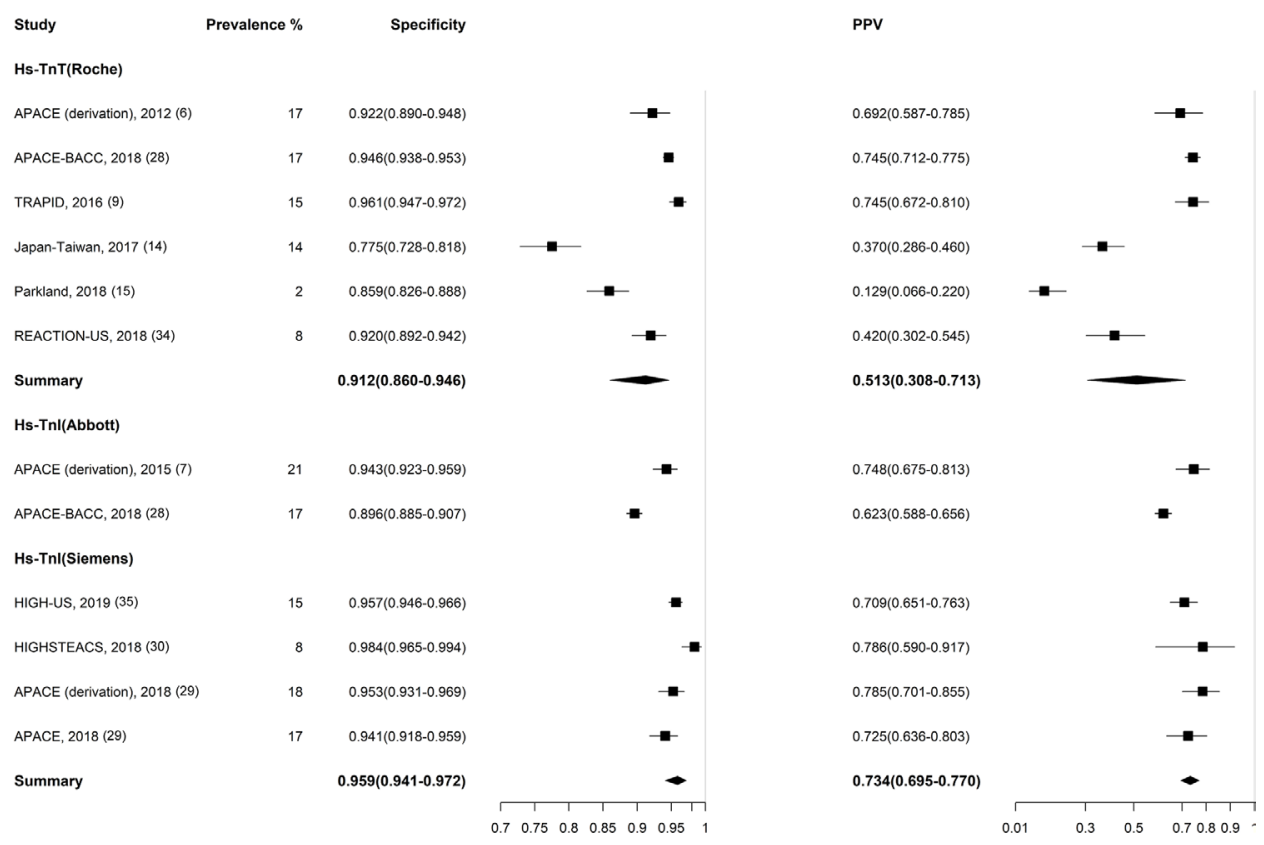

Figure 3 Forest plot for acute myocardial infarction and summary estimates for specificity and PPV. Note: all the three derivation cohorts (Reichlin et $\mathrm{al}^{6}$, Rubini et $\mathrm{al} \mathrm{l}^{7}$ and Boeddinghaus et $\mathrm{al}^{29}$ defined the optimal thresholds based on a classification and regression tree analysis. Boeddinghaus et al (derivation cohort for hs-cTnl (Siemens)) set an optimal threshold for a 70\% PPV. APACE, advantageous predictors of acute coronary syndromes evaluation; BACC, biomarkers in acute cardiac care; HIGHSTEACS, high-sensitivity troponin in the evaluation of patients with acute coronary syndrome; HIGH-US, high-sensitivity cardiac troponin I assays in the United States; PPV, positive predictive value REACTION-US, rapid evaluation of acute myocardial infarction in the United States; TRAPID, the high sensitivity cardiac troponin T assay for rapid rule-out of acute myocardial infarction. 


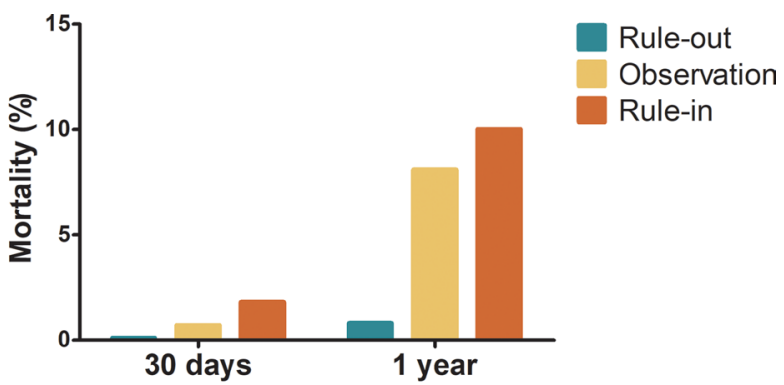

Number of mortality

$\begin{array}{lll}\text { Rule-out } & 3 & 18 \\ \text { Observation } & 8 & 74 \\ \begin{array}{l}\text { Rule-in } \\ \text { Number at risk }\end{array} & 22 & 64 \\ \begin{array}{l}\text { Rule-out } \\ \text { Observation }\end{array} & 2756 & 2312 \\ \text { Rule-in } & 1163 & 935 \\ & 927 & 639\end{array}$

Figure 4 Prognostic outcome of patients in respective triage groups. Data were pooled from three cohorts: APACE, advantageous predictors of acute coronary syndromes evaluation; BACC, biomarkers in acute cardiac care; TRAPID, the high sensitivity cardiac troponin T assay for rapid rule-out of acute myocardial infarction.

performed similarly across different hs-cTn assays. The algorithm ruled in approximately $15 \%$ of the patients with high specificity. A substantial number of patients with unfavourable prognosis were placed in the observation group.

All of the studies in our analysis were prospective, and used the universal definition of myocardial infarction for adjudication of the final diagnosis. Most studies had a low bias towards patient selection. No publication bias was detected. In our sensitivity analysis, we removed derivation studies that would most critically influence the performance of the algorithm. Nevertheless, because many studies used hs-cTn assays as part of the adjudication, or did not use blinding during adjudication, there may be overestimation of the reported accuracy.

In a previous meta-analysis on using hs-cTnT below the limit of detection (LoD) at presentation to rule out AMI, Pickering and associates showed that $30.6 \%$ of patients could be ruled out with a pooled sensitivity of $98.7 \%(95 \% \mathrm{CI}=96.6 \%$ to $99.5 \%) .{ }^{20}$ In our analysis, the addition of a $0-1$ hour change in hs-cTnT levels ruled out more than $50 \%$ of the patients with a pooled sensitivity of $98.4 \%$ (95\% CI=95.1\% to $99.5 \%$ ). The pooled sensitivity of the algorithm was high and comparable across the hs-cTnT (Roche), hs-cTnI (Abbott) and hs-cTnI (Siemens) assays. Nevertheless, the algorithm may not be universally safe if the $1 \%$ miss-rate is desired. ${ }^{37}$ The sensitivity estimate across all assays were less than the consensus goal of $99 \%$. Only five cohorts (APACE, BACC, Japan-Taiwan, Parkland, HIGHSTEACS) reported a sensitivity greater than 99\%. Importantly, two cohorts (Barcelona and Fuwai) reported much lower sensitivities $(<90 \%)$. These observations challenge the universal safety of the algorithm. Given that the pooled sensitivity of the algorithm is below the desired goal of $99 \%$, we suggest that the algorithm be used with caution.

Even though our analysis showed that patients who were ruled out had a favourable short-term and long-term outcome, only three cohorts-mainly cohorts that reported high diagnostic accuracies were represented. Thus, additional investigations are required to ascertain the prognostic outcome of patients ruled out by the algorithm outside of these settings.
Previous systematic reviews that analysed a single 0 -hour sample of hs-cTn above the 99th percentile of a normal reference population to rule in AMI reported the specificity to be $77 \%-82 \% .{ }^{17-19}$ In a recent meta-analysis, using a $50 \mathrm{ng} / \mathrm{L}$ cut-off at presentation to rule in AMI resulted in a pooled specificity of $95 \% .{ }^{22}$ In parallel, the $0 / 1$ hour algorithm could rule in patients at a comparable or a higher specificity of $93.6 \%$. In our analysis, the algorithm based on hs-cTnI (Siemens) had higher specificity compared with that on hs-cTnT (Roche). This could be because the hs-cTnT (Roche) assay has been more extensively investigated in geographically diverse cohorts. Cohorts that reported lower specificities (Japan-Taiwan, Parkland and REACTION-US) using the hs-cTnT (Roche) assay were not investigated using the hs-cTnI (Siemens) assay.

The reported specificities were generally high across the cohorts. Only the Japan-Taiwan cohort ${ }^{14}$ reported a substantially lower specificity $(77.5 \%)$, presumably because patients with chronic renal disease undergoing dialysis were included in this study. However, the PPV for the algorithm was modest at $51.3 \%-73.4 \%$. In this case, it may be helpful to discuss work-up strategies with physicians who are involved in patient management, ${ }^{38}$ because the elevation of troponin often indicates myocardial injury and may require rigorous work-up. ${ }^{39}$

Our study found that the 0/1 hour algorithm places a substantial proportion of patients in the observation zone, for whom management strategy remains unclear. ${ }^{40}$ The rates of MACE and mortality of patients in the observation zone were high at 1 year and comparable to those in the rule-in group. A recent study of 15 international cohorts found that patients who did not have AMI but presented with hs-cTn of more than 10 to $14 \mathrm{ng} / \mathrm{L}-$ similar to patients in the observation zone, had a three times higher risk of death or AMI compared with the general population. ${ }^{41}$ These results suggest that patients that were not ruled by the algorithm carry significant mortality risks. Owing to a lack of individual patient-level data, we were unable to describe the composition of this group of patients. However, a multicentre study showed that patients in the observation zone are typically elderly men with pre-existing coronary artery disease and high long-term mortality. ${ }^{42}$ Clearly, an evidence-based management strategy for this group of patients is urgently needed.

\section{Clinical implications}

Recently, the $0 / 1$ hour algorithm has been demonstrated to produce favourable outcomes in real-world implementation. The introduction of the algorithm shortened time to ED discharge and is associated with low rates of adverse cardiac events and mortality. ${ }^{10-12}$ Our study adds values by demonstrating that the performances of the algorithm based on different hs-cTn assays were comparable. Importantly, this finding may allow institutions to implement hs-cTn testing in patients with suspected AMI, without the financial and logistical challenges of introducing a new assay or analyser exclusively for measuring hs-cTn.

Furthermore, we showed that there are caveats to the algorithm. First, the sensitivity is not universally high across cohorts and may not be sufficiently safe if the $1 \%$ miss-rate for myocardial infarction is desired. Second, a significant number of patients were placed in the observation zone, for whom the longterm prognosis is poor. In consideration of these caveats, it is important to reiterate that this troponin-only algorithm should always be used in conjunction with a detailed clinical assessment and the ECG.

Finally, sampling at the algorithm-defined timings, particularly at 1 hour, may be challenging in some EDs. In these instances, it 
may still be possible to use the $0 / 1$ hour algorithm, as the algorithm has been shown to perform well using samples collected at 2 hours. ${ }^{38}$ However, because most studies in this analysis did not report sampling times, we were unable to provide evidence on the use of the algorithm outside of these pre-defined timings.

\section{Limitations}

There were several limitations to this study. First, we were unable to obtain data of individual patients and could not characterise cases of myocardial infarction missed by the algorithm. Furthermore, the timing of sample collection was not reported in many cohorts. A delayed time from symptom onset to sampling may reduce the potential for missed myocardial infarction and overestimate the algorithm's sensitivity. Second, the substantial risk of bias in the included studies and the inclusion of derivation cohorts in the meta-analysis may influence the overall metaestimate. Because most studies used inclusion and exclusion criteria that precluded certain high risk populations, the accuracy estimates reported in this study may differ from those in the real-world settings. Furthermore, some studies were conducted using samples stored for long periods of time, which could be biased by problems of protein degradation and differences in diagnostic procedures. ${ }^{43} 44$ Third, due to the limited number of studies reporting on patients with chronic kidney disease (CKD), pre-existing coronary artery disease (CAD), or old age, we could not analyse the accuracy of ESC 0/1 hour algorithm on these subgroups. Fourth, because the prevalence of myocardial infarction in our study is substantially higher than that reported by previous epidemiological investigations, ${ }^{45}$ our study may underestimate the NPV and overestimate the PPV. Fifth, some heterogeneity in the accuracy estimates was noted. A lack of relevant contextual information for different studies prevented the use of meta-regression for adjustment. Lastly, in all studies, patients were not managed according to the algorithm, thus troponin levels and differences in clinical management could have influenced the outcomes.

\section{Key messages}

\section{What is already known on this subject?}

- The European Society of Cardiology (ESC) 0/1 hour algorithm has been demonstrated to triage patients with suspected myocardial infarction with high diagnostic accuracy. However, studies evaluating the performance of the algorithm have reported discrepant results.

\section{What might this study add?}

- We performed a systematic review and meta-analysis of studies that investigated on the diagnostic performance of the ESC 0/1 hour algorithm. The algorithm had high sensitivity and specificity across different high-sensitivity cardiac troponin assays. A significant proportion of patients were placed in the observation zone, for whom the long-term prognosis was poor.

\section{How might this impact on clinical practice?}

- Our results support the use of the $0 / 1$ hour algorithm to triage patients with suspected myocardial infarction. However, the algorithm may not be sufficiently safe if the $1 \%$ miss-rate for myocardial infarction is desired. Patients in the observation zone have a poor prognosis and management strategies for these patients are urgently needed.

\section{CONCLUSION}

The ESC 0/1 hour algorithm has high diagnostic accuracy and triage efficacy in ruling out AMI among patients presenting to the ED with symptoms suggestive of myocardial infarction. Different brands of hs-cTn assays show comparable diagnostic accuracy and triage efficacy. A substantial proportion of patients is placed in the observation zone, for whom the prognosis is unfavourable. The algorithm may not be sufficiently safe if the $1 \%$ miss-rate for myocardial infarction is desired. Additional investigations on the implementation of the algorithm will provide more insights regarding its safety and clinical applicability.

\section{Author affiliations}

${ }^{1}$ Department of Medicine, College of Medicine, National Taiwan University, Taipei, Taiwan

${ }^{2}$ Department of Medicine, College of Medicine, Fu Jen Catholic University, Taipei, Taiwan

${ }^{3}$ Department of Internal Medicine III, Cardiology, Angiology and Pneumology, University of Heidelberg, Heidelberg, Germany

${ }^{4}$ Department of Internal Medicine, National Taiwan University Hospital, Taipei, Taiwan ${ }^{5}$ Division of General Internal Medicine, Cedars-Sinai Medical Center, Los Angeles, California, USA

${ }^{6}$ Department of Emergency Medicine, National Taiwan University Hospital, Taipei, Taiwan

${ }^{7}$ Centre for Intelligent Healthcare, National Taiwan University Hospital, Taipei, Taiwan

${ }^{8}$ Health Data Science Research Group, National Taiwan University Hospital, Taipei, Taiwan

Acknowledgements We thank the staff of the Core Labs, Department of Medical Research, National Taiwan University Hospital for their technical support.

Contributors Study concept and design: C-CL and C-HC. Acquisition of data: C-HC, C-HC and GHL. Analysis of data: W-TG and Y-KW. Statistical advice: S-SH and YHY. Drafting of the manuscript: C-CL, C-HC, C-HC and GHL. Critical revision of the manuscript for important intellectual content: W-TG, Y-KW, S-SH, YHY and EG. Study supervision: C-CL and EG.

Funding This work was supported by a research grant from Taiwan National Ministry of Science and Technology Grants MOST 104-2314-B-002 -039 -MY3 and MOST 105-2811-B-002-031.

Competing interests EG reports personal fees from Roche Diagnostics, personal fees from Hoffmann La Roche, personal fees from Brahms Thermo Fischer, personal fees from Daiichi Sankyo, personal fees from Astra Zeneca and personal fees from Bayer Vital outside the submitted work. C-CL reports receiving speaking honorarium from Roche Diagnostics. All other authors report no conflict of interest.

Patient and public involvement Patients and/or the public were not involved in the design, or conduct, or reporting, or dissemination plans of this research.

Patient consent for publication Not required.

Provenance and peer review Not commissioned; externally peer reviewed.

Data availability statement All data relevant to the study are included in the article or uploaded as supplementary information. The authors confirm that the data supporting the findings of this study are available within the article and its supplementary materials.

\section{ORCID iD}

Chien-Chang Lee http://orcid.org/0000-0002-1243-2463

\section{REFERENCES}

1 Niska R, Bhuiya F, Xu J. National Hospital ambulatory medical care survey: 2007 emergency department summary. Natl Health Stat Report 2010:1-31.

2 Goodacre S, Cross E, Arnold J, et al. The health care burden of acute chest pain. Heart 2005;91:229-30.

3 Hess EP, Brison RJ, Perry JJ, et al. Development of a clinical prediction rule for 30-day cardiac events in emergency department patients with chest pain and possible acute coronary syndrome. Ann Emerg Med 2012;59:e111:115-25.

4 Mehta SR, Granger CB, Boden WE, et al. Early versus delayed invasive intervention in acute coronary syndromes. N Eng/ J Med 2009;360:2165-75.

5 Roffi M, Patrono C, Collet JP, et al. Esc guidelines for the management of acute coronary syndromes in patients presenting without persistent ST-segment elevation: Task force for the management of acute coronary syndromes in patients presenting without persistent ST-segment elevation of the European Society of cardiology (ESC). Eur Heart J 2015;2016:267-315 
6 Reichlin T, Schindler C, Drexler B, et al. One-Hour rule-out and rule-in of acute myocardial infarction using high-sensitivity cardiac troponin T. Arch Intern Med 2012;172:1211-8

7 Rubini Gimenez M, Twerenbold R, Jaeger C, et al. One-Hour rule-in and rule-out of acute myocardial infarction using high-sensitivity cardiac troponin I. Am J Med 2015; 128:e864:861-70

8 Reichlin T, Twerenbold R, Wildi K, et al. Prospective validation of a 1-hour algorithm to rule-out and rule-in acute myocardial infarction using a high-sensitivity cardiac troponin T assay. CMAJ 2015;187:E243-52.

9 Mueller C, Giannitsis E, Christ M, et al. Multicenter evaluation of a 0-Hour/1-Hour algorithm in the diagnosis of myocardial infarction with high-sensitivity cardiac troponin T presented at the European Society of cardiology annual meeting, September 2014, Barcelona, Spain. Ann Emerg Med;2016:76-87.

10 Twerenbold R, Costabel JP, Nestelberger T, et al. Outcome of applying the ESC 0/1 hour algorithm in patients with suspected myocardial infarction. J Am Coll Cardiol 2019; 74:483-94.

11 Stoyanov KM, Hund H, Biener M, et al. RAPID-CPU: a prospective study on implementation of the ESC 0/1-hour algorithm and safety of discharge after rule-out of myocardial infarction. Eur Heart J Acute Cardiovasc Care 2020;9:39-51.

12 Chew DP, Lambrakis K, Blyth A, et al. A randomized trial of a 1-hour troponin T protocol in suspected acute coronary syndromes: the rapid assessment of possible acute coronary syndrome in the emergency department with high-sensitivity troponin T study (RAPID-TnT). Circulation 2019;140:1543-56.

13 Anand A, Shah ASV, Beshiri A, et al. Global adoption of high-sensitivity cardiac troponins and the universal definition of myocardial infarction. Clin Chem 2019:65:484-9.

14 Shiozaki M, Inoue K, Suwa S, et al. Utility of the 0-hour/1-hour high-sensitivity cardiac troponin T algorithm in Asian patients with suspected non-ST elevation myocardial infarction. Int J Cardiol 2017:249:32-5.

15 Vigen R, Kutscher P, Fernandez F, et al. Evaluation of a novel Rule-Out myocardia infarction protocol incorporating high-sensitivity troponin T in a US Hospital. Circulation 2018;138:2061-3.

16 Duran-Cambra A, Alquezar A, Garcia-Osuna A, et al. A new cardiac troponin I assay with enhanced detection limit improves the rapid rule-out of acute myocardial infarction. European Heart Journal: Acute Cardiovascular Care 2018;7:144.

17 Sethi A, Bajaj A, Malhotra G, et al. Diagnostic accuracy of sensitive or high-sensitive troponin on presentation for myocardial infarction: a meta-analysis and systematic review. Vasc Health Risk Manag 2014;10:435-50.

18 Lipinski MJ, Baker NC, Escárcega RO, et al. Comparison of conventional and highsensitivity troponin in patients with chest pain: a collaborative meta-analysis. Am Heart J 2015;169:e16:6-16.

19 Zhelev Z, Hyde C, Youngman E, et al. Diagnostic accuracy of single baseline measurement of Elecsys troponin T high-sensitive assay for diagnosis of acute myocardial infarction in emergency department: systematic review and meta-analysis. BMJ 2015;350:h15.

20 Pickering JW, Than MP, Cullen L, et al. Rapid Rule-out of acute myocardial infarction with a single high-sensitivity cardiac troponin T measurement below the limit of detection: a collaborative meta-analysis. Ann Intern Med 2017;166:715-24.

21 Chapman AR, Lee KK, McAllister DA, et al. Association of high-sensitivity cardiac troponin I concentration with cardiac outcomes in patients with suspected acute coronary syndrome. JAMA 2017:318:1913-24.

22 Arslan M, Dedic A, Boersma E, et al. Serial high-sensitivity cardiac troponin T measurements to rule out acute myocardial infarction and a single high baseline measurement for swift rule-in: a systematic review and meta-analysis. Eur Heart J Acute Cardiovasc Care 2019;2048872618819421.

23 Mclnnes MDF, Moher D, Thombs BD, et al. Preferred reporting items for a systematic review and meta-analysis of diagnostic test accuracy studies: the PRISMA-DTA statement. JAMA 2018;319:388-96.
24 Stroup DF, Berlin JA, Morton SC, et al. Meta-Analysis of observational studies in epidemiology: a proposal for reporting. meta-analysis of observational studies in epidemiology (moose) group. JAMA 2000;283:2008-12.

25 Thygesen K, Alpert JS, Jaffe AS, et al. Third universal definition of myocardial infarction. Eur Heart J 2012;33:2551-67.

26 Whiting PF, Rutjes AWS, Westwood ME, et al. QUADAS-2: a revised tool for the quality assessment of diagnostic accuracy studies. Ann Intern Med 2011;155:529-36.

27 Deeks JJ, Macaskill P, Irwig L. The performance of tests of publication bias and other sample size effects in systematic reviews of diagnostic test accuracy was assessed. $J$ Clin Epidemiol 2005; 58:882-93.

28 Twerenbold R, Neumann JT, Sörensen NA, et al. Prospective validation of the 0/1-h algorithm for early diagnosis of myocardial infarction. J Am Coll Cardiol 2018;72:620-32.

29 Boeddinghaus J, Twerenbold R, Nestelberger T, et al. Clinical validation of a novel high-sensitivity cardiac troponin I assay for early diagnosis of acute myocardial infarction. Clin Chem 2018;64:1347-60.

30 Chapman AR, Fujisawa T, Lee KK, et al. Novel high-sensitivity cardiac troponin I assay in patients with suspected acute coronary syndrome. Heart 2019;105:616.

31 Chapman AR, Hesse K, Andrews J, et al. High-Sensitivity cardiac troponin I and clinical risk scores in patients with suspected acute coronary syndrome. Circulation 2018;138:1654-65.

32 Ruangsomboon O, Mekavuthikul P, Chakorn T, et al. The feasibility of the 1-H highsensitivity cardiac troponin T algorithm to rule-in and rule-out acute myocardial infarction in Thai emergency patients: an observational study. Int J Emerg Med 2018;11:43

33 Mokhtari A, Borna C, Gilje P, et al. A 1-h Combination Algorithm Allows Fast Rule-Out and Rule-In of Major Adverse Cardiac Events. J Am Coll Cardiol 2016;67:1531-40.

34 Gandolfo CM, McCord J, Hudson MP, et al. Rapid evaluation of acute myocardial infarction using a change in high-sensitivity cardiac troponin T over 1 hour. Circulation 2018;136.

35 Nowak RM, McCord J, Christenson R, et al. Performance of a novel high sensitivity cardiac troponin I assay for a one hour algorithm for evaluation of non ST segment elevation myocardial infarction in the United States. J Am Coll Cardiol 2019;73:135.

36 Lin Y, Zhang G, Feng G, et al. 1/3 hours rule in and rule out algorithm for NSTEM using a high-sensitivity cardiac troponin I at emergency department in Chinese population. Clin Chem 2018;64:S49.

37 Than $\mathrm{M}$, Herbert $\mathrm{M}$, Flaws $\mathrm{D}$, et al. What is an acceptable risk of major adverse cardiac event in chest pain patients soon after discharge from the emergency department?: a clinical survey. Int J Cardiol 2013;166:752-4.

38 Pickering JW, Greenslade JH, Cullen L, et al. Assessment of the European Society of cardiology 0-Hour/1-Hour algorithm to Rule-Out and Rule-In acute myocardia infarction. Circulation 2016;134:1532-41.

39 Park KC, Gaze DC, Collinson PO, et al. Cardiac troponins: from myocardial infarction to chronic disease. Cardiovasc Res 2017:113:1708-18.

40 Twerenbold R, Boeddinghaus J, Nestelberger T, et al. Clinical use of high-sensitivity cardiac troponin in patients with suspected myocardial infarction. J Am Coll Cardiol 2017;70:996-1012.

41 Neumann JT, Twerenbold R, Ojeda F, et al. Application of high-sensitivity troponin in suspected myocardial infarction. N Eng/ J Med 2019;380:2529-40.

42 Nestelberger T, Wildi K, Boeddinghaus J, et al. Characterization of the observe zone of the ESC 2015 high-sensitivity cardiac troponin 0h/1h-algorithm for the early diagnosis of acute myocardial infarction. Int J Cardiol 2016;207:238-45.

43 Agarwal SK, Avery CL, Ballantyne CM, et al. Sources of variability in measurements of cardiac troponin T in a community-based sample: the Atherosclerosis risk in Communities study. Clin Chem 2011;57:891-7.

44 Herman DS, Kavsak PA, Greene DN. Variability and error in cardiac troponin testing: an ACLPS critical review. Am J Clin Pathol 2017;148:281-95.

45 Kohn MA, Kwan E, Gupta M, et al. Prevalence of acute myocardial infarction and other serious diagnoses in patients presenting to an urban emergency department with chest pain. J Emerg Med 2005;29:383-90. 